\title{
Neuromodulation of Bilateral Hippocampal Foci, an Alternative for Mesial Temporal Lobe Seizures in Patients with Non-Lesional MRI: Long-Term Follow-up
}

\author{
Velasco $F^{1}$, Vázquez-Barrón $D^{2}$, Pérez-Pérez $D^{3}$, Cuéllar-Herrera $M^{1}$, Trejo $D^{1}$, Montes de Oca $M^{2}$ and Velasco $A L^{4 *}$ \\ ${ }^{1}$ Epilepsy Clinic, Stereotactical and Functional Neurosurgery Unit. Eduardo Liceaga General Hospital of Mexico, Mexico \\ ${ }^{2}$ PhD Program Medical School, National Autonomous University of Mexico, Mexico \\ 3 PECEM program, National Autonomous University of Mexico, Mexico \\ ${ }^{4}$ Head of the Epilepsy Clinic, Stereotactical and Functional Neurosurgery Unit. Eduardo Liceaga General Hospital of Mexico, Mexico
}

*Corresponding author: Dr. Ana Luisa Velasco, Head of the Epilepsy Clinic, Stereotactical and Functional Neurosurgery Unit. Eduardo Liceaga General Hospital of Mexico, Mexico, Tel: 0445554097631; E-mail: analuisav@yahoo.com

Received date: Sep 23, 2016; Accepted date: Oct 12, 2016; Published date: Oct 16, 2016

Copyright: $\odot 2016$ Velasco F, et al. This is an open-access article distributed under the terms of the Creative Commons Attribution License, which permits unrestricted use, distribution, and reproduction in any medium, provided the original author and source are credited.

\section{Abstract}

Background: Patients with bilateral independent hippocampal refractory seizures, who show no evidence of lesion in the MRI, constitute a challenge for their treatment. The risk of severe retrograde amnesia and/or residual seizures is high when bilateral temporal lobectomy is performed. Several groups have reported electrical neuromodulation of the hippocampus as a reversible surgical alternative for mesial temporal lobe epilepsy seizures that are refractory to medical treatment.

Methods: In the Epilepsy Clinic of the General Hospital we have had three patients with bilateral independent foci and non-lesional MRI. The present publication shows their long-term seizure status and their neuropsychological outcome. Stimulation parameters were $3.0 \mathrm{~V}$ amplitude, high frequency $(130 \mathrm{~Hz}), 450 \mu$ s pulse width, one minute ON and 4 min OFF cyclic stimulation. Follow-up extended over 9 years.

Results: Patients showed dramatic seizure reduction. In two patients in whom stimulation was stopped (due to battery depletion or skin erosion), seizure reduction persisted for months after stimulation stopped, but eventually seizures reappeared slowly. One patient has had uninterrupted stimulation and has been seizure free for nine years. The neuropsychological tests showed no functional decrease at the selected modulation parameters.

Conclusion: Bilateral hippocampal stimulation is effective in seizure control in patients in whom ablative surgery is not recommended.

\section{Introduction}

The death of Henry Molaison (HM) in December 2008, kindled the discussion based on the postmortem analysis of one of the most emblematic cases in epilepsy surgery [1]. This patient had bilateral mesial temporal lobe seizures that were refractory to several antiepileptic medications. In an attempt to control his seizures, at age 27, a bilateral temporal lobectomy with amygdalo-hippocampectomy was performed. Since this moment on, his life changed [2]. Even though his seizures were mostly controlled by the procedure, the surgery rendered severe anterograde amnesia.

Numerous neuropsychological, imaging and anatomic studies were performed [3]. The conclusion of these studies indicates that bilateral temporal lobectomy is a high-risk surgery due to its neurological consequence. Even though patients with refractory mesial temporal lobe seizures are candidates for temporal lobectomy [4,5] patients are rejected for ablative surgery if they have bilateral independent hippocampal epileptic foci [6]. The prognosis is worsened if there is no evidence of hippocampal sclerosis in the MRI [7]. Refractory bilateral seizures are a challenge for every epileptologist, neurologist and neurosurgeon.
In 2000 our group proposed electrical stimulation as a feasible alternative [8] for mesial temporal lobe epilepsy and other authors have confirmed the positive results of this method [9-13]. Since our initial publications no neuropsychological deficit has been observed at low amplitude stimulation parameters, other investigators have confirmed this finding [14-16]. Herein we present a long-term followup in seizure control and neuropshychogical outcome of three patients who had no evidence of hippocampal sclerosis in the MRI and showed bilateral independent epileptic foci evidenced by intracranial recordings.

\section{Methods}

The hippocampal focus stimulation protocol was approved by the Research and Ethics Committees of the General Hospital of Mexico. Nine patients were included initially. In the present paper we present the long-term outcome of three patients from this group, those who had bilateral independent hippocampal foci demonstrated by depth electrode recordings. The three patients had bilateral hippocampal stimulation directed to the epileptic foci. Two males and one female are described, ages 44, 21 and 24 years old, respectively. Complex 
Citation: Velasco F, Vázquez-Barrón D, Pérez-Pérez D, Cuéllar-Herrera M, Trejo D, Montes de Oca M, Velasco AL (2016) Neuromodulation of Bilateral Hippocampal Foci, an Alternative for Mesial Temporal Lobe Seizures in Patients with Non-Lesional MRI: Long-Term Follow-up. Epilepsy J 2: 116. doi:10.4172/2472-0895.1000116

Page 2 of 5

partial seizures with secondary generalization started at 3,12 and 11 years, respectively.

Surface EEG showed bilateral temporal spikes and MRIs did not demonstrate hippocampal lesion. Neuropsychological battery was performed including the Neuropsi Attention and Memory Battery [17-18]. This is a comprehensive battery developed for evaluating attention, memory, and executive functions. The tests have been standardized and validated in Spanish-speaking subjects from 6 to 85 years of age.

The results were corrected according to age and education. Subtests are organized into summary index counts that yield a Total Attention and Memory Index score, as well as separate ones for Attention and Memory Processes. One patient had mild verbal memory impairment and two had normal tests. Batteries were applied in the baseline phase, 6 and 12 months with neuromodulation and once a year afterwards.

In an attempt to determine the precise location and the laterality of the epileptic foci, patients were included in phase II invasive study protocol. Octopolar $\mathrm{AD}$-Tech [Racine $\mathrm{WN}$ ] depth electrodes were implanted through occipital burr holes (Figure 1A).

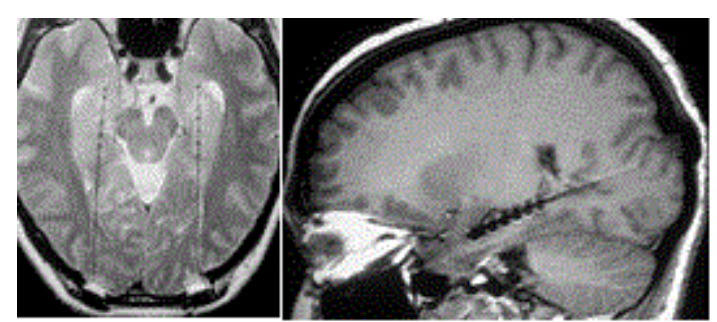

Figure 1(A): Depth electrodes were implanted through occipital burr holes.

To direct the trajectory of the electrodes, we used the Praezis 3.0 Plus program using pre operatory MRI. During implantation we performed fluoroscopic control and images of final site were taken. Once implanted, patients underwent MRI to verify correct implantation and afterwards, antiepileptic medication was tapered and continuous video EEG monitoring was performed until several spontaneous seizures arising independently from right and left anterior hippocampus were recorded, confirming bilateral foci (Figure 1B and 1C).

Patients were informed of their results and invited to participate in the neuromodulation protocol. Diagnostic electrodes were removed and substituted by 3387 DBS by Medtronic Inc. Minneapolis, MN. Again, we used Praezis 3.0 Plus, planning the trajectory of therapeutic electrodes based on preoperative MRI and diagnostic electrode position with post implantation MRI. They were directed so that 3 contacts of the DBS electrode were within the area where the epileptic foci were localized.

Fluoroscopic control was undertaken during implantation, the trajectory and location of the DBS electrodes to the fluoroscopic scans of the diagnostic electrodes were compared. MRI scans were obtained again to confirm target and afterwards the pulse generator (IPG_Itrel 3 by Medtronic) was connected. Stimulation parameters were set as follows: $3.0 \mathrm{~V}, 130 \mathrm{~Hz}, 450 \mu \mathrm{s}$, cycling $1 \mathrm{~min}$ ON and $4 \mathrm{~min}$ OFF. All patients were OFF stimulation during the first month to discard implantation effect and were enrolled in a double blind study during the first three months. This report includes the open label phase of all patients.

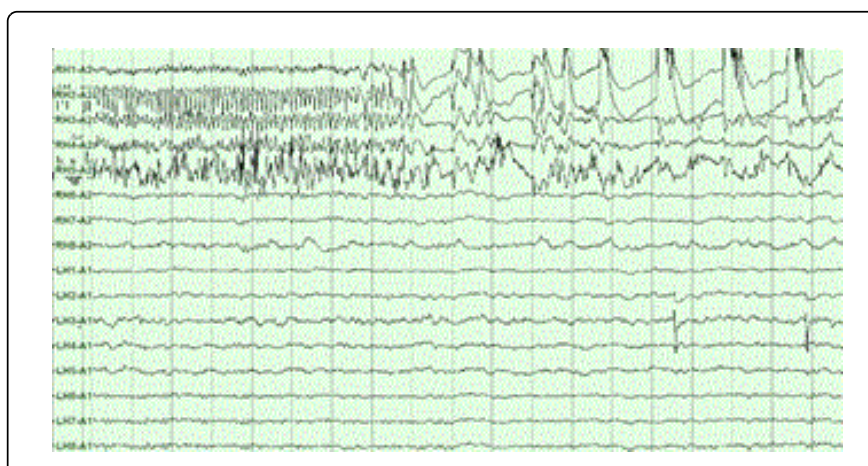

Figure 1(B): Patient's 1 right hippocampal seizure onset.

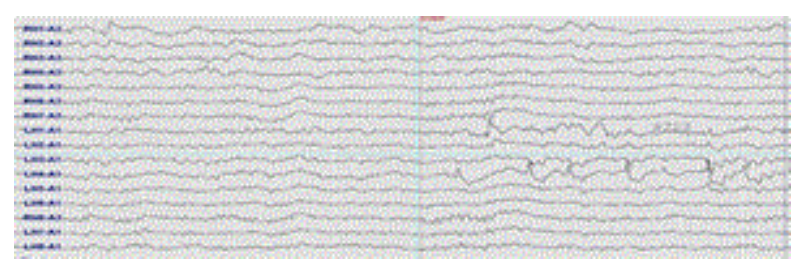

Figure 1(C): Patient's 1 left hippocampal seizure onset.

\section{Results}

\section{Stimulating contacts position}

Figure 2 shows stimulated contact position in each patient. Note that the electrodes were directed to the epileptic foci, not a specific structure. Patient 1 had right contact in dentate gyrus and left one in CA1. Patient 2 had both right and left contacts in dentate gyrus and patient 3 had right contact located in parahippocampus and left one in subiculum. The long-term follow-up was 120 to 167 months.

\section{Seizure decrease}

Figure 3 shows the seizure count graphs for each patient. Patient 1 was the first bilateral case selected for chronic stimulation. As such we did not know if bilateral stimulation would cause neuropsychological problems. This is why initially he was stimulated on the right hippocampal focus during 6 months. He had 50\% seizure decrease. On month 7 , bilateral stimulation started and seizures continued decreasing until he became seizure free during 9 months. At the end of month 29, he had 3 complex partial in a single day due to battery depletion. Pulse generators were changed. He remained with one seizure every 3 months from month 15 till month 71 . On month 72 he had one complex partial seizure with secondary generalization due to battery depletion and was not able to afford pulse generators again. Seizures have increased progressively, but never reached basal numbers. 
Citation: Velasco F, Vázquez-Barrón D, Pérez-Pérez D, Cuéllar-Herrera M, Trejo D, Montes de Oca M, Velasco AL (2016) Neuromodulation of Bilateral Hippocampal Foci, an Alternative for Mesial Temporal Lobe Seizures in Patients with Non-Lesional MRI: Long-Term Follow-up. Epilepsy J 2: 116. doi:10.4172/2472-0895.1000116

Page 3 of 5
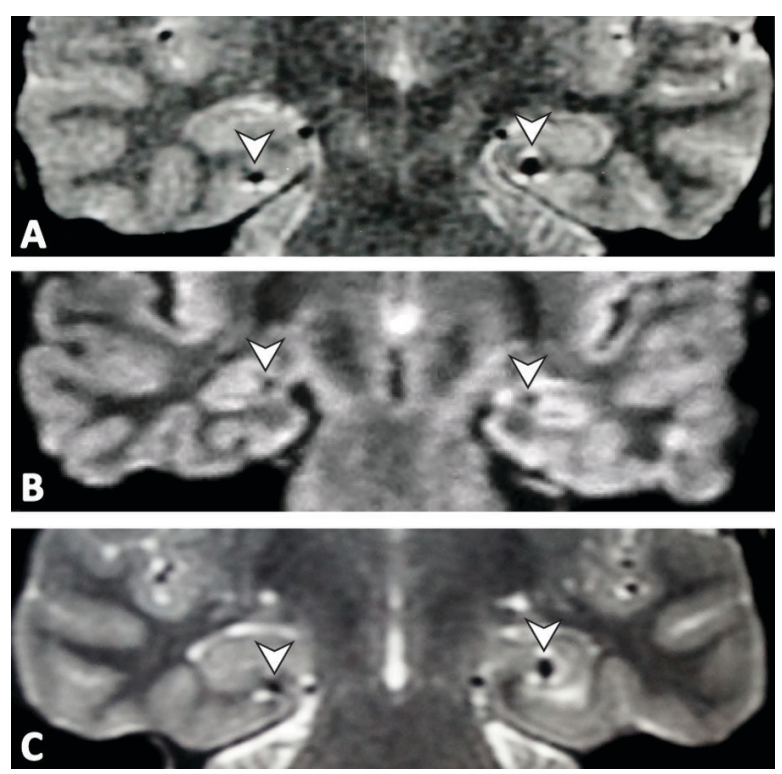

Figure 2: MR1 T2 weighted showing bilateral stimulated contacts position in each patient.

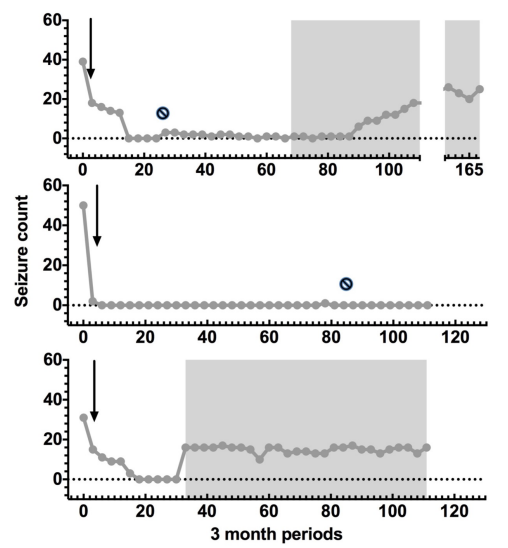

Figure 3: Graphs of the seizure counts every 3 months. Arrows show the month when stimulation is turned ON. All patients had a decrease in their seizure counts during stimulation periods and never returned to basal counts after stimulation showed its positive effect, regardless if the batteries continued working. Crossed circles show when batteries were replaced. Shadow indicates time when patient was not stimulated.

Patient 2 had a dramatic seizure decrease during the first three months of stimulation and remained seizure free till month 81 when three complex partial seizures appeared due to battery depletion. Pulse generators were changed immediately and patient has remained seizure free.

Patient 3 had a progressive seizure decrease during 18 months and afterwards she was seizure free. On month 31 she had to be explanted due to skin erosion. Since then seizures increased but never returned to baseline numbers remaining $50 \%$ reduction.

\section{Neuropsychological testing}

Table 1 shows the three patients' results comparing baseline grades with 1 year of neuromodulation. Note that none of the patients had a decrease in their performance, they either stayed the same or improved compared to baseline.

Figure 4 neuropsychological results of patient 1 at two different measurements: baseline and 2 years of stimulation. Note that the patient showed improvement in almost all skills during stimulation compared to baseline. Neuropsychological testing grades of patient 2 were good before and during stimulation, but he improved specifically in verbal memory and this is important since he has had a very successful development, graduating as Master in Foreign Languages at the University, speaks fluently German, English and French. Patient 3 maintained her neuropsychological performance in same levels.

\section{Discussion}

Several groups have performed hippocampus stimulation and have found a beneficial result in seizure reduction. Compared to other stimulation targets it has a better outcome. Vagal stimulation reduces seizures in best cases 45\% [19]. Responsive stimulation shows a progressive seizure reduction that takes several years to show $45 \%$ seizure reduction [20]. In our previously published studies, Patients with no MRI evidence of mesial temporal sclerosis had a $>95 \%$ seizure reduction. This decrease in seizures takes 1 to 2 months. The difference between the responsive stimulation and our open loop cyclic mode can be explained due to difficulty in having a reliable seizure prediction system. Currently there are a number of groups working on seizure detection and their results could improve the results of the responsive stimulation mode [21-24].

All patients reported herein, had seizure reduction. It has been suggested that the intra hippocampal electrode insertion per se, results in seizure remission $[25,26]$. This study shows that seizure control happened in different times: two (patient 2), fifteen (patient 1) and eighteen months (patient 3 ). The reason for the differential response to DBS is unknown, but delay in seizure reduction indicates that antiepileptic effect was not secondary to micro lesion produced by implantation of recording and DBS electrodes. The fact that seizure control depended on DBS and was not the result of spontaneous remission is clearly illustrated in Figure 3, where one can see that battery depletion or explantation was associated with recurrence of seizures.

However, seizure recurrence did not occur abruptly but progressively increased over a period of months (Case 2) to over a year and a half (Case 1), which indicates that the antiepileptic effect extends beyond DBS, as has been described in cerebellar [27] and thalamic [28] stimulation. This stresses the importance that studies using double blind phases place the maneuver at the beginning of chronic stimulation and avoid cross over. 
Citation: Velasco F, Vázquez-Barrón D, Pérez-Pérez D, Cuéllar-Herrera M, Trejo D, Montes de Oca M, Velasco AL (2016) Neuromodulation of Bilateral Hippocampal Foci, an Alternative for Mesial Temporal Lobe Seizures in Patients with Non-Lesional MRI: Long-Term Follow-up. Epilepsy J 2: 116. doi:10.4172/2472-0895.1000116

Page 4 of 5

\begin{tabular}{|c|c|c|c|c|c|c|c|c|c|}
\hline Patient & $\begin{array}{l}\text { Education } \\
\text { level }\end{array}$ & Basal performance & $\begin{array}{l}\text { Attention } \\
\text { memory }\end{array}$ & $\begin{array}{l}\text { Verbal } \\
\text { memory }\end{array}$ & $\begin{array}{l}\text { Digit } \\
\text { counting }\end{array}$ & $\begin{array}{l}\text { Logic } \\
\text { memory }\end{array}$ & $\begin{array}{l}\text { Visual } \\
\text { reproduction }\end{array}$ & Wind mill & Bezares \\
\hline 1 & Elementary & Poor & No changes & Improved & improved & $\begin{array}{l}\text { No } \\
\text { changes }\end{array}$ & Improved & Improved & Improved \\
\hline 2 & University & $\begin{array}{l}\text { Good } \\
\text { percentiles }\end{array}$ & Improved & Improved & improved & Improved & No changes & $\begin{array}{l}\text { No } \\
\text { changes }\end{array}$ & No changes \\
\hline 3 & $\begin{array}{l}\text { Technical } \\
\text { career }\end{array}$ & $\begin{array}{ll}\text { Good } & \text { lower } \\
\text { percentiles } & \end{array}$ & No changes & $\begin{array}{l}\text { No } \\
\text { changes }\end{array}$ & $\begin{array}{l}\text { No } \\
\text { changes }\end{array}$ & $\begin{array}{l}\text { No } \\
\text { changes }\end{array}$ & No changes & $\begin{array}{l}\text { No } \\
\text { changes }\end{array}$ & No changes \\
\hline
\end{tabular}

Table 1: The three patients' results comparing baseline grades with 1 year of neuromodulation.

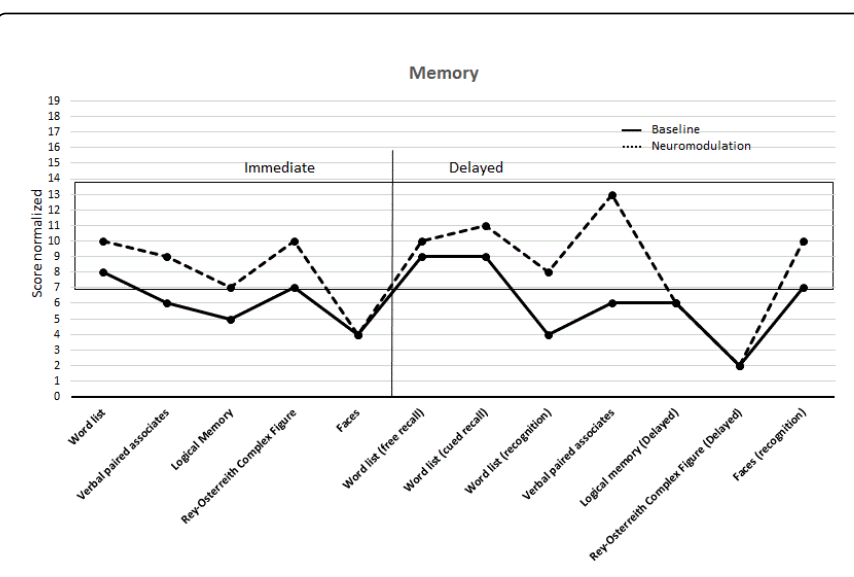

Figure 4: Neuropsychological results of patient 1 at two different measurements: baseline and 2 years of stimulation.

Bilateral Anterior Temporal Lobectomies (ATL) produces severe memory deficit In contrast, bilateral Hippocampal Electrical Stimulation (HES) did not induce neuropsychological deterioration in our cases, Moreover, in our case 3, posterior HES in the right side was accompanied by normalization of a mild deficit for non-verbal memory one year after the onset of stimulation, as reported by Boex [29]. In this case improvement in memory occurred when monthly seizures had decreased by $80 \%$ and in Patient 1 memory improvement was detected when patient was seizure free. Case 2 had normal memory performance and remained so. Therefore, bilateral simultaneous HES for seizure control does not seem to interfere with memory function and might enhance memory, as reported by Boex [29]. Neuropsychological improvement has been reported with centromedian thalamic stimulation in cases with Lennox-Gastaut and clearly relates to seizure control and normalization of the EEG background [30-32].

There has been discussion regarding the precise stimulation target. Our group has suggested that it should be directed to the epileptic focus and others to the subiculum [33]. In this report patient 1 had both stimulating contacts positioned in the area corresponding to the epileptic foci [32]. Patient 2 had both contacts very near the epileptic focus in the dentate gyrus. Patient 3, who took the longest time to achieve seizure freedom (18 months), had one of the contacts in the subiculum and the other in the para hippocampus.

Two of the patients had skin erosions over the skullcap used to hold DBS electrodes (Case 1) and the connector between DBS and extension cable (Case 3) in the retro auricular area. This type of complications has been described previously [34]. Unfortunately neuromodulation could not be prolonged due to economic reasons.

\section{Conclusion}

Stimulation of the hippocampus epileptic foci is effective in controlling mesial temporal lobe seizures; it does not interfere with memory process when applied bilaterally. For the moment being, we report 3 cases, but in the future it may become a less invasive alternative for refractory cases. The efficacy is still related to some factors that are not fully known, such as the most effective place of stimulation in the hippocampal formation and optimization of stimulation parameters. Future work in the improvement of hardware for this indication should include single recording-stimulation electrodes with an increase in number of contacts, spaced to cover the entire hippocampus so that the patient can undergo a single surgical implantation to localize the epileptic focus and afterwards stimulate it. This would reduce costs and risks. And to avoid the system rejection for epileptic patients, the length of DBS electrodes should be reduced.

\section{References}

1. Annese J, Schenker-Ahmed NM, Bartsch H, MaechlesP, Sheh C, et al. (2014) Postmortem examination of patient HM.s brain based on histological sectioning and digital 3D reconstruction. Nat Commun 3: $1-9$.

2. Corkin S [2002] What's new with the amnesic patient HM? Nat Rev 3: 153-160.

3. Corkin S, Amaral DG, González RG, Johnson DA, Hyman BT et al. (1997) H.M's medial temporal lobe lesion: findings from magnetic resonance imaging. J Neurosc 17: 3964-3979

4. Kwan P, Brodie MJ (2000) Early identification of refractory epilepsy. N Engl J Med 342: 314-319.

5. Kwan P, Sperling MR (2009) Refractory seizures: try additional antiepileptic drugs [after two have failed] or go directly to early surgery evaluation? Epilepsia 8: 57-62.

6. Jehi L (2015) The risk-benefit ratio for Tempoal lobe resection in patients with bilateral mesial temporal lobe epilepsy. Epilepsy Curr 15: 78-69M.

7. Bell M, Rao S, So E, Trenerry M, Kazemi N, et al. (2009) Epilepsy surgery outcomes in temporal lobe epilepsy with a normal MRI. Epilepsia 50: 2053-2060.

8. Velasco F, Velasco M, Velasco AL (2000) Subacute electrical stimulation of the hippocampus blocks intractable temporal lobe seizures and paroxysmal EEG activities. Epilepsia 41: 158-169.

9. Boon P, Vonck K, De Herdt V (2007) Deep brain stimulation in patients with refractory temporal lobe epilepsy. Epilepsia 48: 1551-1560. 
Citation: Velasco F, Vázquez-Barrón D, Pérez-Pérez D, Cuéllar-Herrera M, Trejo D, Montes de Oca M, Velasco AL (2016) Neuromodulation of Bilateral Hippocampal Foci, an Alternative for Mesial Temporal Lobe Seizures in Patients with Non-Lesional MRI: Long-Term Follow-up. Epilepsy J 2: 116. doi:10.4172/2472-0895.1000116

Page 5 of 5

10. Velasco AL, Velasco F, Velasco M (2007) Electrical stimulation of the hippocampal epileptic foci for seizure control: a double blind, long term follow-up study. Epilepsia 48: 1895-1903.

11. Vonck K, Sprengers M, Carrette E, Miatton M, Meurs A, et al. (2013) A decade of experience with deep brain stimulation for patients with refractory medial temporal lobe epilepsy. Int J Neural Syst 23: 1-13.

12. Vonck K, Boon P, Achtedn E (2002) Long-term amygdalohippocampal stimulation for refractory temporal lobe epilepsy. Ann Neurol 52: 556-565.

13. Cukiert A, Cukiert M, Burattini JA (2014) Seizure outcome after hippocampal deep brain stimulation in a prospective cohort of patients with refractory temporal lobe epilepsy. Seizure 23: 6-9.

14. McLachland RS, Pigott S, Tellez-Zenteno JF (2010) Bilateral hippocampal stimulation for intractable temporal lobe epilepsy: Impact on seizures and memory. Epilepsia 51: 304-307.

15. Milner B (1975) Psychological aspects of focal epilepsy and its neurosurgical management. In Purpura DP (ed) Neurosurgical Management of the Epilepsies. Raven Press, New York pp: 299-321.

16. Helmstaedter C, Petzol I, Blen GC (2011) The cognitive consequence of resecting non-lesional tissues in epilepsy surgery--Results from MRI and histopathology negative patients with temporal lobe epilepsy. Epilepsia 52: 1402-1408.

17. Ostrosky-Solís F, Gómez ME, Matute E (2003) Neuropsi Atención y Memoria, manual, instructivo y puntuaciones totales. Mexico, American Book Store pp: 7-75.

18. Ostrosky-Solis F, Ardila A, Roselli M (1999) Neuropsi a brief neuropsychological test battery in Spanish with norms by age and educational level. J Int Neuropsychol Soc 5: 413-433.

19. Mollet L, Raedt R, Delbeke J, Tahry R, Grimonprez A, et al. (2013) Electrophysiological responses from vagus nerve stimulation in rats. Int J Neural Syst 23: 6-1350027.

20. Fisher B, Velasco AL (2014) Neurostimulation in the treatment of Epilepsy. Nature Rev Neurol 10: 261-270.

21. Alexandridis A (2013) Evolving RBF Neural Networks for Adaptive SoftSensor Design. Int J Neural Syst 23: 6.

22. Ghosh-Dastidar S, Adeli H (2009) A new supervised learning algorithm for multiple spiking neural Networks and application in epilepsy and seizure detection. Neural Netw 2009: 1419-1431.
23. Osorio I (2014) Automated Seizure Detection Using EKG. Int J Neural Syst 24: 2-1450001.

24. Sharma P, Khan YU, Farooq O, Tripathi M, Adeli H (2014) Waveletstatistical features approach for non-convulsive seizure detection. Clin EEG Neurosci 274: 284.

25. Schulze-Bonhage A, Denning D, Wagner K (2010) Seizure control resulting from intrahippocampal depth electrode insertion. J Neurol Neurosurg Psychiatry 81: 352-353.

26. Katariwala NM, Bakay RA, Pennell PB (2001) Remission of intractable partial epilepsy following implantation of intracranial electrodes. Neurology 57: 1505-1507.

27. Velasco F, JD Carrillo-Ruiz, F Brito, Velasco M, Velasco AL, et al. (2005) Double blind, randomized controlled pilot study of bilateral cerebellar stimulation for treatment of intractable motor seizures. Epilepsia 46: 1071-1081.

28. Davis R (2000) Cerebellar stimulation for cerebral palsy spasticity, function and seizures. Arch Med Res 31: 290-299.

29. Velasco F, Velasco M, Jiménez FF, Velasco AL, Rojas B, et al. (2002) Centromedian nucleus stimulation for epilepsy: Clinical, electroencephalographic, and behavioral observations. Thalamus Relat Syst 1: 387-398.

30. Boex C, Seeck M, Vulliemoz S (2011) Chronic deep brain stimulation in mesial temporal lobe epilepsy. Seizure 20: 485-490.

31. Velasco AL, Velasco F, Jimenez F, Velasco M, G. Castro, et al. (2006) Neuromodulation of the centromedian thalamic nuclei in the treatment of generalized seizures and improvement of quality of life in patients with Lennox-Gastaut syndrome. Epilepsia 47: 1203-1212.

32. Valentin A, Gracía-Navarrete E and Chelvarajah R (2010) Deep brain stimulation of the centromedian thalamic nucleus for the treatment of generalized and frontal epilepsies. Epilepsia 54: 1823-1833.

33. Bondallaz P, Boex C, Rossetti AO (2013) Electrode location and clinical outcome in hippocampal electrical stimulation for mesial temporal lobe epilepsy. Seizure 22: 390-395.

34. Oh MY, Abosch A, Kim SH (2002) Long-term hardware-related complications of deep brain stimulation. Neurosurgery 50: 1268-1274. 